\title{
Clinicopathological Compatibility Rates with Regard to Skin Punch Biopsies
}

Nuran SÜNGÜ ${ }^{1}$, Hayriye T. DOĞAN ${ }^{1}$, Aydan KILIÇARSLAN ${ }^{1}$, Huban S.O. YAVUZ², Seda MARALI ${ }^{2}$, Merve KIRAN $^{1}$, Akın AKTAŞ3 ${ }^{3}$ Ahmet METIN³

\footnotetext{
${ }^{1}$ Department of Pathology, Ankara Yıldırım Beyazit University, Faculty of Medicine, Atatürk Education and Research Hospital, Ankara, Turkey. ${ }^{2}$ Department of Pathology, Atatürk Education and Research Hospital, Ankara, Turkey.

${ }^{3}$ Department of Dermatology, Ankara Yıldırım Beyazit University, Faculty of Medicine, Atatürk Education and Research Hospital, Ankara, Turkey.
}

\section{ABSTRACT}

The aim of this study was to explore the compatibility of clinical prediagnosis and histopathological diagnosis of skin punch biopsies at the Pathology Department of Ankara Atatürk Training and Research Hospital, Yıldirim Beyazıt University, Turkey. Several factors may change the appearance of a typical lesion because the skin is open to the external environment, and clinical diagnoses may be difficult. As a result, the histopathological assessment of skin punch biopsies and the support given by clinicians to the pathologist are extremely important.

Whole punch biopsy diagnoses, which were evaluated at the department between the years 2006 and 2015, were grouped according to the inflammatory reaction patterns determined and described in Weedon's Textbook of Dermatopathology. Subsequently, the compatibility rates of clinical prediagnosis were studied, focusing especially on the first three prediagnoses. A total of 2967 punch biopsies were evaluated at the department between the years 2006 and 2015; $93.09 \%$ were diagnosed with inflammatory dermatosis, and $6.9 \%$ with tumors. The compatibility rate between clinical prediagnosis and pathological diagnosis was $85.77 \%$; $65.75 \%$ of the cases were compatible with the first three prediagnoses. The compatibility rate of inflammatory dermatosis was 93.09\%. The most common compatibility with prediagnosis was seen in epidermal maturation and keratinization and elastic tissue lesions with a rate of $100 \%$.

Although performing a skin biopsy is easy, histopathological evaluation of the skin has not been used commonly. In fact, it is recommended that skin biopsy should be performed in cases with possible neoplasia, bullous diseases, or dermatosis, which have not been clinically diagnosed. In these cases, clinicopathological correlation is important to reach a true diagnosis. This study aimed to determine mutual contributions of clinicians and pathologists by evaluating clinicopathological compatibility rates.

Key Words: Clinicopathological compatibility rates, punch biopsies,skin

\section{INTRODUCTION}

The typical signs of dermatological diseases have various causes. Moreover, the same etiological factors may lead to different kinds of eruptions. Hence, it may be difficult to comment on the clinical signs. Pathological examination supports the clinical data in such cases (1).

Clinical data are important in certain cases of inflammatory dermatosis because their histopathological appearances are similar. Especially the diagnosis of inflammatory dermatosis is primarily dependent on clinical data (1).

For these reasons, dermatologists and pathologists should work in coordination with each other. If the dermatologist has knowledge about basic dermatopathological patterns, such as psoriasis and pityriasis rubra pilaris when psoriasiform dermatitis is mentioned, compatibility increases. Similarly, if a pathologist has knowledge about dermatosis with pustular lesions or vesiculobullous lesions, compatibility increases. 


\begin{tabular}{|c|c|c|}
\hline & Patien & ר) $\%$ \\
\hline \multicolumn{3}{|l|}{ Gender } \\
\hline Male & 1308 & 44.08 \\
\hline Female & 1659 & 55.9 \\
\hline \multicolumn{3}{|l|}{ Compliance with diagnosis } \\
\hline Preliminary diagnosis & 2545 & 85.77 \\
\hline The first three diagnosis & 1951 & 65.75 \\
\hline $\begin{array}{l}\text { Tumors } \\
\text { Preliminary diagnosis }\end{array}$ & 205 & 6.90 \\
\hline Preliminary diagnosis & 152 & 74.14 \\
\hline The first three diagnosis & 124 & 60.48 \\
\hline Inflammatory dermatosis & 2762 & 93.09 \\
\hline Preliminary diagnosis & 2393 & 86.64 \\
\hline The first three diagnosis & 1827 & 66.14 \\
\hline
\end{tabular}

This study aimed to determine the age, gender, and clinical prediagnosis of patients who had undergone a punch biopsy at dermatology outpatient clinics and also to evaluate the correlation between these prediagnoses and histopathological diagnoses.

\section{MATERIALS AND METHODS}

The diagnoses and clinical data of 2967 punch biopsies, which were analyzed at the Pathology Department of Ankara Atatürk Training and Research Hospital, Yıldırım Beyazıt University, Turkey, between the years 2006 and 2015, were retrospectively evaluated from hospital archives. Pathological diagnoses were grouped according to the inflammatory reaction patterns determined and described in Weedon's Textbook of Dermatopathology (Table 1). The diseases were grouped according to their pathological diagnoses; inflammatory dermatosis; their lichenoid reaction pattern (interface dermatitis) (lichen planus, lichen planus variants, lichen niditus, lichen striatus, lichenoid drug reactions, fixed drug reactions, erythema multiforme, lupus erythematosus and variants, lichen sclerosus et atrophicus, pityriasis lichenoides, and so on); their psoriasiform reaction pattern (psoriasis, pityriasis rubra pilaris, lichen simplex chronicus, and so on.); their spongiotic reaction pattern (allergic and irritant contact dermatitis, atopic dermatitis, pityriasis rosea, seborrheic dermatitis, nummular eczema, stasis dermatitis, and so on); their vesiculobullous reaction pattern (intracorneal, subcorneal, intraepidermal, suprabasillar, and subepidermal blisters); their granulomatous reaction pattern (sarcoidal, tuberculoid, necrobiotic. and so on.); their vasculopathic reaction pattern (urticarias, acute vasculitis, eosinophilic vasculitis, granuloma faciale, neutrophilic dermatosis, Sweet's syndrome, chronic lymphocytic vasculitis, pigmented purpuric dermatosis, pityriasis lichenoides, erythema annulare centrifugum, perniosis, pyoderma gangrenosum, and so on); disorders of epidermal maturation and keratinization (palmoplantar keratoderma and other genodermatosis); disorders of pigmentation (hyper- and hypopigmentation disorders); disorders of collagen (scleroderma, sclerodermoid disorders, perforating collagenoses, and other hypertrophic collagenoses); disorders of elastic tissue; cutaneous deposits (amyloidosis, calcinosis, bone and cartilage, and so on); panniculitis; diseases of cutaneous appendages (folliculitis, alopecias, pilosebaceous and apocrine disorders); reactions to physical agents (polymorphic light eruption, phototoxic dermatitis); cutaneous drug reactions; disorders of papillomatosis; infections; infestations; and tumors. Then, the compatibility rates of clinical prediagnosis were researched in the pathology reports focusing especially on the first three prediagnoses. If no correlation existed between the pathology results and prediagnoses, it was determined as incompatible. Also, the compatibility rates between the two pathologists interested in dermatopathology and all the other pathologists in the department were evaluated.

\section{RESULTS}

A total of 2967 punch biopsies were evaluated at the pathology department between the years 2006 and 2015; 2762 (93.09\%) were diagnosed with inflammatory dermatosis and 205 (6.9\%) with tumors. Also, 55.9\% of these cases were female, and $44.8 \%$ were male (Table 1). Their mean age was 44 years. Almost half of these cases were evaluated by the pathologists interested in dermatopathology. Spongiotic dermatosis was noted most commonly in 675 cases (22.75\%) inflammatory dermatosis and benign tumors were seen most commonly in 117 cases (3.9\%) (Table 2). The compatibility rate between clinical prediagnosis and pathological diagnosis was $85.77 \%$; $65.75 \%$ of the cases were compatible with the first three prediagnoses. The compatibility rate of inflammatory dermatosis was $93.09 \%$. The most common compatibility with prediagnosis was seen in epidermal maturation and keratinization (8/8) and elastic tissue lesions (2/2) at a rate of $100 \%$. The most common compatibility with the first three prediagnoses was seen in alopecia at $84.82 \%$ and amyloidosis at $83.33 \%$. The least common compatibility with prediagnosis was seen in infections and infestations at $68.85 \%$ and folliculitis at $70.93 \%$. The least common compatibility with the first three prediagnoses was seen in cutaneous deposits at 50\% (2/4) and elastic tissue 
TABLE 2: Compliance rates with the diagnosis and the first three preliminary diagnosis of tumors.

\begin{tabular}{llll}
\hline & $\begin{array}{l}\text { Pathological diagnosis } \\
\mathrm{n} / 205\end{array}$ & $\begin{array}{l}\text { The first three diagnosis } \\
\mathrm{n} / 205\end{array}$ & $\begin{array}{l}\text { Total } \\
\mathrm{n} / 2967\end{array}$ \\
\hline Tumors & $152(74.14 \%)$ & $124(60.48 \%)$ & $205(6,9 \%)$ \\
Benign Tumors & $84(40,97 \%)$ & $67(32,68 \%)$ & $117(3,9 \%)$ \\
Malignant Tumors & $68(33,17 \%)$ & $57(27,80 \%)$ & $88(2,9 \%)$ \\
\hline
\end{tabular}

lesions at 50\% (1/2) (Table 3). The compatibility rate of cases evaluated by the pathologists interested in dermatopathology was $77.9 \%$, and the compatibility rate of the cases evaluated by the other pathologists was $77.6 \%$. The compatibility rate with the first three prediagnoses was found to be $71.6 \%$ in cases evaluated by the pathologists interested in dermatopathology and $47.9 \%$ when evaluated by the others.

\section{DISCUSSION}

Histopathological examination is the gold standard diagnostic method in dermatology. Biopsy is avoided for cosmetic reasons in certain cases such as pediatric patients and patients with lesions on facial regions (2). In fact, a biopsy is performed to diagnose, stage the disease, and evaluate the response of treatment (3).

TABLE 3: Compliance rate with pathological diagnosis and the first three diagnosis are listed in the table.

\begin{tabular}{|c|c|c|c|}
\hline Reactions pattern & Pathological diagnosis & The first three diagnosis & Total \\
\hline The lichenoid reaction pattern & $366(88,55 \%)$ & $270(64,59 \%)$ & $418(100 \%)$ \\
\hline The psoriasiform reaction pattern & $406(94,19 \%)$ & $313(72,45 \%)$ & $431(100 \%)$ \\
\hline The spongiotic reaction pattern & $586(86,81 \%)$ & $458(67,85 \%)$ & $675(100 \%)$ \\
\hline The vesiculobullous reaction pattern & $31(81,57 \%)$ & $24(63,15 \%)$ & $38(100 \%)$ \\
\hline The granulomatous reaction pattern & $53(92,98 \%)$ & $36(63,15)$ & $57(100 \%)$ \\
\hline The vasculopathic reaction pattern & $281(91,53 \%)$ & $205(66,77 \%)$ & $307(100 \%)$ \\
\hline Disorders of epidermal maturation and keratinization & $8(100 \%)$ & $5(62,5 \%)$ & $8(100 \%)$ \\
\hline Disorders of pigmentation & $84(83,16 \%)$ & $63(62,37 \%)$ & $101(100 \%)$ \\
\hline Disorders of collogen & $116(95,08 \%)$ & $84(68,85 \%)$ & $122(100 \%)$ \\
\hline Disorders of elastic tissue & $2(100 \%)$ & $1(50 \%)$ & $2(100 \%)$ \\
\hline Cutaneous deposits(Amiloidosis) & $17(94,44 \%)$ & $15(83,33 \%)$ & $18(100 \%)$ \\
\hline Diseases of cutaneous appendages(Folliculitis) & $61(70,93 \%)$ & $52(60,46 \%)$ & $86(100 \%)$ \\
\hline Diseases of cutaneous appendages(Alopecias) & $110(98,21 \%)$ & $95(84,82 \%)$ & $112(100 \%)$ \\
\hline Panniculitis & $27(93,10 \%)$ & $24(82,75 \%)$ & $29(100 \%)$ \\
\hline Cutaneous drug reactions & $101(92,66 \%)$ & $74(67,88 \%)$ & $109(100 \%)$ \\
\hline Reactions to physical agents & $27(96,42 \%)$ & $20(71,42 \%)$ & $28(100 \%)$ \\
\hline İnfections and infestations & $42(68,85 \%)$ & $31(50,81 \%)$ & $61(100 \%)$ \\
\hline Tumors & $152(74,14 \%)$ & $124(60,48 \%)$ & $205(100 \%)$ \\
\hline Cutaneous infiltrates non lymphoid & $10(100 \%)$ & $7(70,0 \%)$ & $10(100 \%)$ \\
\hline Disorders of papillomatosis & $12(85,71 \%)$ & $8(57,14 \%)$ & $14(100 \%)$ \\
\hline Cutaneous deposits & $3(75,0 \%)$ & $2 / 50,0 \%)$ & $4(100 \%)$ \\
\hline Other & $80(60,60 \%)$ & $60(45,45 \%)$ & $132(100 \%)$ \\
\hline Total & $2545(85,77 \%)$ & $1951(65,75 \%)$ & $2967(100 \%)$ \\
\hline
\end{tabular}


Inflammatory dermatoses can be categorized into six major groups according to their reaction patterns in the histopathological examination: lichenoid, psoriasiform, spongiotic, vesiculobullous, granulomatous, and vasculopathic patterns (1). This study aimed to explore the clinicopathological compatibility rates by classifying the histopathological diagnoses according to their reaction patterns.

In this study, 2545 (85.77\%) of 2976 cases were compatible with clinical prediagnosis, and 1951 (65.75\%) cases were compatible with the first three diagnoses. The study by Çalka et al. on 460 skin biopsies showed that $87.2 \%$ of cases were compatible with clinical prediagnosis and $67.6 \%$ of cases were compatible with the first three diagnoses (4).

The study by Sula et al. involving 239 cases demonstrated that $74.4 \%$ of the pathological diagnoses were compatible with clinical preliminary diagnosis and $67.7 \%$ of cases were compatible with the first three diagnoses (3).

The study by Dilek et al. involving 1047 cases showed that the clinicopathological compatibility rate with clinical preliminary diagnosis was $78.1 \%$ and the rate with the first three diagnoses was $73.6 \%(5)$.

The present results were also compatible with the available literature.

Hosamane et al. found a clinicopathological correlation in $45.45 \%$ cases of psoriasis, $48.71 \%$ cases of lichen planus, and $66.67 \%$ cases of pityriasis rosea in their study on noninfectious erythematous papulosquamous skin lesions (6). The compatibility rate was found to be $94.19 \%$ in psoriasiform dermatitis and $86.81 \%$ in spongiotic dermatitis. The present values were found to be higher because the compatibility rates of histopathological patterns were explored.

Roh et al. found incompatibility in $24.2 \%$ cases of 206 seborrhoeic keratoses (7). The present study found incompatibility in $25.86 \%$ of tumor cases.

Dermatologists and pathologists should work in concordance with each other to reach an accurate diagnosis in dermatopathology. Dermatologists should send a short summary of clinic anamnesis along with the biopsy material to the pathology department. Sellheyer et al. investigated clinicopathological compatibility rates among 4451 cases, including dermatologists and nondermatologists. Inflammatory skin diseases were correctly diagnosed in $71 \%$ of cases by dermatologists and only $34 \%$ of cases by nondermatologists. The overall accuracy of clinical diagnosis depends heavily on the clinicopathological correlation. Without sufficient clinical data, histopathological diagnosis is or restricted. This review concluded that without basic knowledge of dermatology, the clinicopathological correlation was compromised (8).

Besides the differences between dermatologists and other clinicians regarding clinicopathological compatibility, differences also exist between pathologists and non-dermatopathologists. The present study found that the compatibility rate of cases evaluated by pathologists interested in dermatopathology was $77.9 \%$ and the compatibility rate of the cases evaluated by the other pathologists was $77.6 \%$. The compatibility rate with the first three prediagnoses was $71.6 \%$ in the cases evaluated by the pathologists interested in dermatopathology but only $47.9 \%$ in those evaluated by other pathologists. The compatibility rates with the first three prediagnoses were found to be higher as far as the pathologists interested in dermatopathology were concerned. In conclusion, it is thought that clinical data from dermatologists helps the pathologist to reach a more accurate diagnosis and increase clinicopathological compatibility. Furthermore, the active interest of pathologists in dermatopathology also contributes positively in this situation.

\section{REFERENCES}

1. David Weedon. An approach to the interpretation of skin biopsies. Weedon's Skin Pathology. Printed in China, Elsevier, p 4, 2010

2. Garg P, Kaur T, Malhotra SK and Amarjit. Study of the Dermoscopic Findings and Their Correlation with Histopathological Findings in Various Lichenoid Dermatoses. J Clin Exp Dermatol Res 2015, 6:6.

3. Sula $B$, Uçak $H$, Arıca $M$, Türkçü G. Yetişkin hastalarda deri biyopsilerinin klinik özellikleri ve klinikopatolojik korelasyonu. Abant Medical Journal 2015; 4(3):251-254.

4. Çalka Ö, Akdeniz N, Kösem M, Bayram İ, Metin A, Uğraş S. Deri hastalıkların tanısında histopatolojinin yeri. Tıp Araştırmaları Dergisi 2005:3(1):27-30.

5. Dilek N, Saral Y, Çobanoğlu B. Dermatozlarda Klinik ve Histopatolojik Korelasyon. Fırat Üniversitesi Sağlık Bilimleri Tıp Dergisi 2009;23(2):81-84.

6. Hosamane S, Pai M, Philipose TR, Nayarmoole U. Clinicopathological Study of Non-Infectious Erythaematous Papulosquamous Skin Diseases. J Clin Diagn Res 2016:10(6):EC19-22.

7. Roh NK, Hahn HJ, Lee YW, Choe YB, Ahn KJ. Clinical and Histopathological Investigation of Seborrheic Keratosis. Ann Dermatol 2016;28(2):152-8

8. Klaus Sellheyer, Wilma F. Bergfeld. A retrospective biopsy study of the clinical diagnostic accuracy of common skin diseases by different specialties compared with dermatology. Journal of the American Academy of Dermatology 2005;52(5):823-830. 